\title{
Reversibilidade da Cardiomiopatia Dilatada na Síndrome de Sheehan: Um Relato de Caso
}

\author{
Dilated Cardiomyopathy Reversibility in Sheehan's Syndrome: A Case Report
}

\author{
Maria Luíza Barbosa Fernandes Dourado, ${ }^{1 \oplus}$ Taís Paiva da Costa, ${ }^{1}$ Márcia Sampaio de Carvalho, ${ }^{1}$ Carlos Geraldo \\ Guerreiro de Moura ${ }^{1,2}$ \\ Obras Sociais Irmã Dulce, ${ }^{1}$ Salvador, BA - Brasil \\ Escola Bahiana de Medicina e Saúde Pública, ${ }^{2}$ Salvador, BA - Brasil
}

\section{Introdução}

A síndrome de Sheehan, descoberta em 1937 por Harold Leeming Sheehan, é descrita como pan-hipopituitarismo secundário à necrose hipofisária após hemorragia pós-parto. ${ }^{1}$ A apresentação do quadro clínico depende da deficiência hormonal apresentada, e pode envolver alterações nos níveis de cortisol sérico, função tireoidiana, hormônios do crescimento, amamentação e hormônios sexuais. ${ }^{2}$ Embora escassamente descrita na literatura, há relatos de miocardiopatia dilatada associada à síndrome de Sheehan, alguns com reversibilidade da cardiopatia após terapia de reposição hormonal. ${ }^{3}$ Este trabalho relata um caso clínico de síndrome de Sheehan associada à miocardiopatia dilatada que apresentou melhora da função cardíaca após terapia de reposição hormonal.

\section{Relato de Caso}

O presente artigo é sobre uma mulher de 36 anos, casada, do lar, natural de Inhambupe/BA, que havia sido hospitalizada em serviço médico terciário com dispneia progressiva há 2 meses que evoluiu para dispneia em repouso 02 dias após a hospitalização. Além disso, ela relatou edema de membros inferiores e edema periorbital. Também se queixava de náuseas e vômitos pós-prandiais por 01 semana com restos de comida, sem muco ou sangue, afebril. Referia internação anterior, aos 18 anos, devido a complicações decorrentes de pré-eclâmpsia e hemorragia pós-parto, negou transfusões de sangue. Também descreveu agalactia e amenorreia pós-parto. A paciente tem uma vida sexual ativa com um único parceiro e não utiliza nenhum método contraceptivo. Ao exame físico, estado geral regular, com fala confusa, hipoatividade e hipotensão ( $\triangle \mathrm{PAS} 100-80 \mathrm{mmHg} \times \triangle \mathrm{PAD}$

\section{Palavras-chave}

Hipopituitarismo; Síndrome de Sheehan; Cardiomiopatia Dilatada; Diagnóstico por Imagem; Terapia de Reposição Hormonal.

Correspondência: Maria Luíza Barbosa Fernandes Dourado •

Obras Sociais Irmã Dulce - Avenida Dendezeiros do Bonfim, 161. CEP

40420-415, Bonfim, Salvador, BA - Brasil

E-mail: mlbfdourado@gmail.com

Artigo recebido em 14/08/2019, revisado em 22/12/2019, aceito em 09/03/2020

DOI: https://doi.org/10.36660/abc.20190547
70-50 mmHg). Pele com turgor e elasticidade reduzidos, presença de edema periorbital. O sistema cardiovascular apresentava precórdio calmo, impulso apical não palpável e não visível, sons cardíacos hipofonéticos, sem sopros, sem som cardíaco extra. Extremidades com perfusão, com edema depressível $+1 / 4+$, frio, indolor. Outros acompanhamentos sem alterações.

Os exames laboratoriais iniciais mostraram TSH inadequadamente normal em 4,93 $\mu \mathrm{UI} / \mathrm{mL}(0,38-5,3)$ com T4 livre abaixo de $0,4 \mathrm{ng} / \mathrm{dL}(0,5-1,2)$, hiponatremia normovolêmica (sódio 133mEq/ L - VR 136-144). Exames complementares na hospitalização: hemoglobina $12 \mathrm{mg} / \mathrm{dL}$, hematócrito 35,9\%, leucograma 12.880: 89\% segmentados, $4 \%$ linfócitos, $1 \%$ eosinófilos e $6 \%$ monócitos; plaquetas $165.000 / \mathrm{mm}^{3}$ e função renal normal.

A reposição de hidrocortisona em bolus de $500 \mathrm{mg}$ foi seguida de levotiroxina em baixas doses $(50 \mathrm{mcg} /$ dia). Após introdução da terapia hormonal, a paciente apresentou melhora da hipoatividade e astenia apresentada na hospitalização.

O quadro clínico e a resposta à hormonioterapia confirmaram a suspeita diagnóstica de hipopituitarismo secundário à necrose hipofisária após hemorragia pós-parto, confirmado pelos seguintes testes: GH 0,1 ng/mL $(0,5-3,6)$, beta-estradiol $20 \mathrm{pg} / \mathrm{mL}$ (<40 pós-menopausa), FSH 4,5 $\mathrm{mUI} / \mathrm{mL}$ (16 - 113: pós-menopausa), LH 2,96 mIU/mL (10,8 - 58,6: pós-menopausa), prolactina 3,36 ng/mL (2-15), ACTH 35,8 pg/mL (VR 7,2-63,3) e cortisol sérico matinal 1,5 mcg/dL (5,4-25). A ressonância nuclear magnética cerebral revelou sela túrcica vazia parcial, com herniação da cisterna supra-selar para o interior da sela túrcica, identificando uma fina camada de glândula pituitária no assoalho selar, com realce homogêneo ao meio de contraste (Figura 1).

Ciente do quadro de dispneia e hipofonia dos sons cardíacos associado à síndrome edemigênica, foram solicitadas radiografia de tórax (Figura 2A) e ecocardiografia transtorácica. A radiografia mostrou a presença de cardiomegalia. O ecocardiograma mostrou miocardiopatia dilatada com disfunção sistólica ventricular esquerda significativa, às custas de hipocinesia difusa, fração de ejeção do ventrículo esquerdo (FEVE) de $27 \%$ e regurgitação mitral leve.

Uma vez realizados os ajustes da hormonioterapia instituída com levotiroxina $100 \mathrm{mcg} / \mathrm{dia}$ e prednisona $10 \mathrm{mg} / \mathrm{dia}$, houve melhora clínica e radiológica significativa (Figura 2B). O ecocardiograma serial após 2 semanas de tratamento mostrou melhora de $12 \%$ na fração de ejeção 


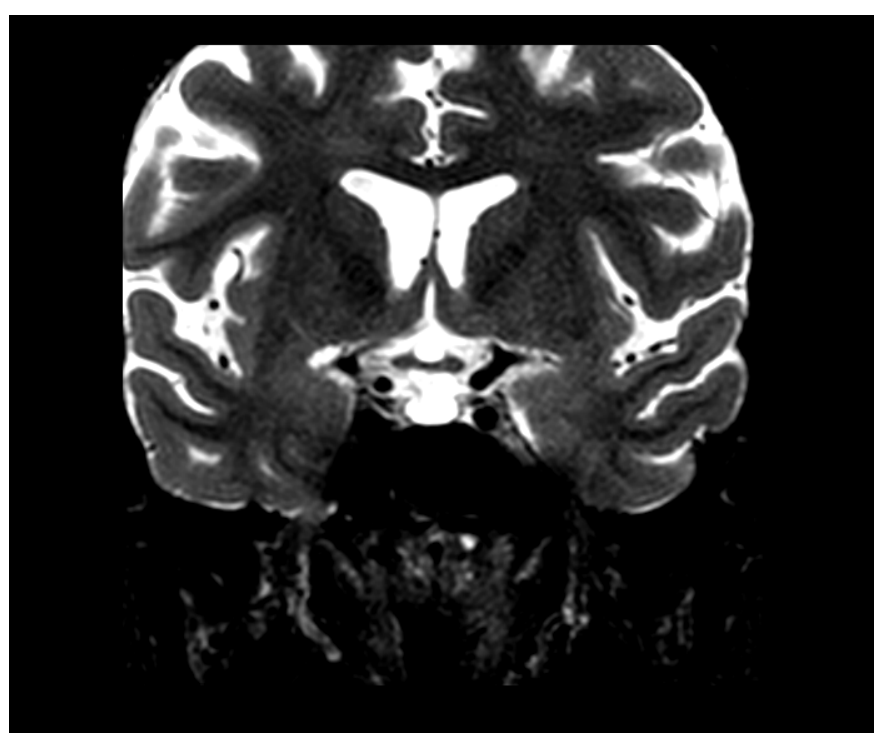

Figura 1 - Ressonância magnética da sela túrcica parcialmente vazia.

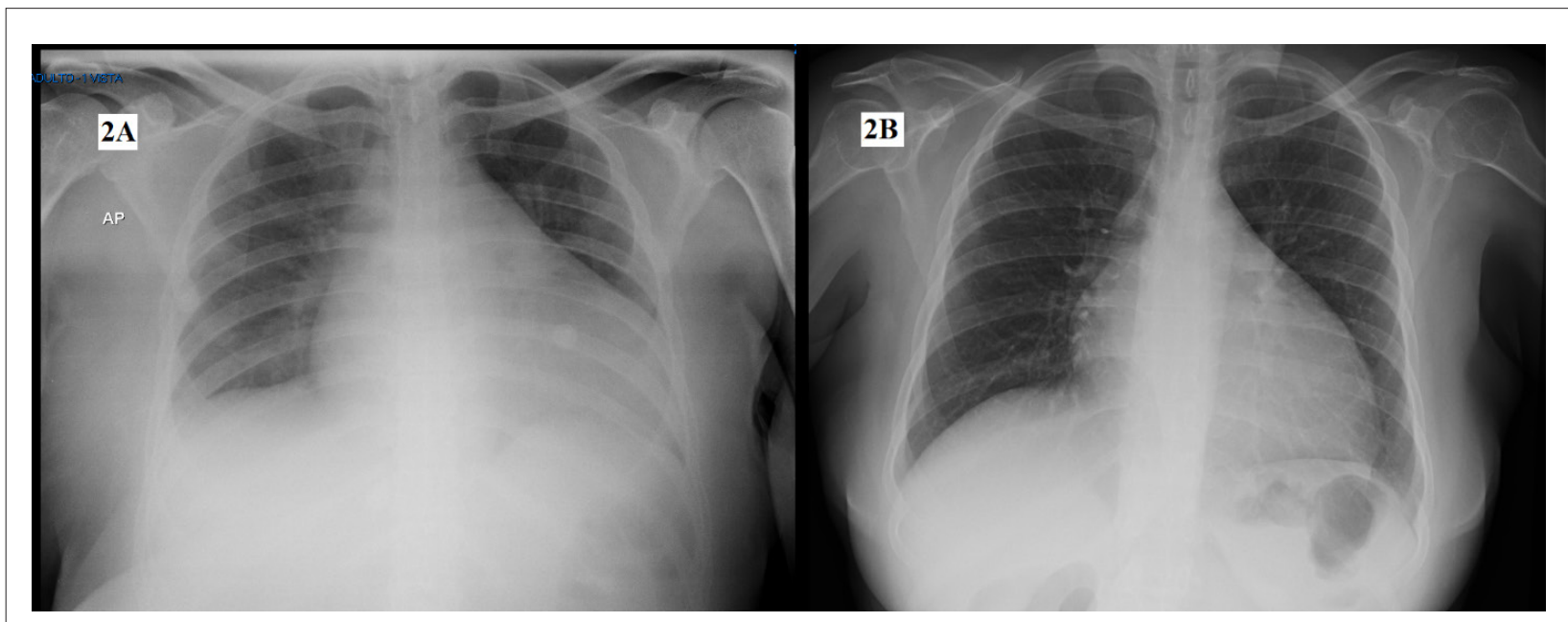

Figura 2-A) Radiografia de tórax na hospitalização. B) Radiografia de tórax após terapia hormonal.

e redução da disfunção sistólica global, mesmo sem terapia específica para Insuficiência Cardíaca Congestiva (ICC).

A terapia para insuficiência cardíaca com redução da fração de ejeção só foi introduzida uma semana antes da alta hospitalar, pois até o momento a paciente apresentava níveis pressóricos limítrofes. Ela foi encaminhada ao ambulatório de cardiologia, com orientações sobre o uso de betabloqueadores cardiosseletivos, além de espironolactona.

\section{Discussão}

A incidência da síndrome de Sheehan, secundária à hemorragia periparto, está diretamente relacionada à qualidade do atendimento médico durante a gravidez. ${ }^{4}$ A mortalidade materna é um importante marcador do estado de saúde da população. Uma das principais causas de mortalidade materna é a hemorragia pós-parto, que pode ter como consequência a ocorrência de necrose hipofisária. ${ }^{5}$

A apresentação clínica da síndrome de Sheehan depende do nível de isquemia da glândula pituitária. ${ }^{1}$ Cerca de $75 \%$ das células da hipófise precisam estar comprometidas para causar deficiência hormonal secundária. ${ }^{3}$ Os sinais e sintomas são divididos em doença aguda e crônica. ${ }^{6}$ Os sintomas agudos consistem em hipotensão, choque, taquicardia, hipoglicemia, hiponatremia, fadiga extrema, náuseas e vômitos, classicamente representados por insuficiência adrenal aguda. Cronicamente, os pacientes 
podem apresentar astenia, fadiga, diminuição da força muscular, constipação, intolerância ao frio relacionada ao hipotireoidismo central; redução da libido, agalactia, amenorreia e infertilidade, devido à redução do estímulo gonadotrófico; incluindo transtornos psiquiátricos. ${ }^{1}$

Segundo a literatura, a busca por atendimento médico é motivada por distúrbios hidroeletrolíticos, principalmente a hiponatremia. Durante os primeiros atendimentos à paciente do caso, náuseas, vômitos e hiponatremia direcionaram a abordagem diagnóstica e terapêutica. Só foi possível saber a história obstétrica da paciente após a resolução do estado confusional. Porém, no contexto de emergência, a paciente foi internada com síndrome edemigênica de etiologia cardíaca.

A apresentação da Síndrome de Sheehan como ICC é atípica e o envolvimento cardíaco foi considerado o mais raro entre os descritos. ${ }^{7}$ Em 2013, Doshi et al., ${ }^{3}$ já haviam mencionado a entidade hipo-poliglandular associada à reversibilidade da função cardíaca. A cardiopatia do paciente com hipotireoidismo está associada principalmente ao derrame pericárdico, quando o tempo de enchimento ventricular é reduzido, podendo resultar em tamponamento cardíaco. ${ }^{8}$ Quando relacionada à insuficiência adrenal, é relatada em pacientes com hipocortisolismo como parte das síndromes poliglandulares autoimunes do tipo 1, também reversíveis após correção hormonal. ${ }^{9}$ No entanto, a etiologia da miocardiopatia dilatada relacionada à síndrome de Sheehan permanece desconhecida.

A melhora da função ventricular demonstrada no quadro clínico durante o curto período de duas semanas foi peculiar. Houve um aumento da fração de ejeção de $27 \%$ para 39\% após duas semanas de hospitalização, apesar do uso de terapia formal para insuficiência cardíaca. Outros casos descritos na literatura expõem a reversibilidade da miocardiopatia dilatada quando associada à síndrome de Sheehan; porém, a maioria deles associando reposição hormonal e terapia direcionada à ICC com fração de ejeção reduzida. ${ }^{7,9-12}$

Doshi et al., ${ }^{3}$ abordaram o caso clínico de uma paciente de 42 anos com uma apresentação de emergência de choque cardiogênico secundário a pan-hipopituitarismo devido à síndrome de Sheehan. Amenorreica há 14 anos (data da última gestação), a paciente foi tratada com glicocorticoide, levotiroxina e 48 horas de uso de inotrópico. Seis meses após o início da terapia, a paciente apresentou aumento de 100\% da FEVE (inicial: 20\%, seguimento: $40 \%$ ), melhora dos parâmetros radiológicos e tornou-se assintomática. Em 2014, na Arábia Saudita, foi estudado o caso de uma jovem paciente que deu entrada no pronto-socorro apresentando dispneia e com síndrome edemigênica há 6 meses com diagnóstico de miocardiopatia dilatada periparto. Porém, após extensa investigação, o diagnóstico inicial foi reconsiderado, pois a paciente apresentava história de hemorragia periparto, insuficiência adrenal e tireoidiana, além de sela túrcica vazia. Assim, ela foi diagnosticada com síndrome de Sheehan associada à miocardiopatia dilatada, revertida em 06 meses após a reposição hormonal. ${ }^{7}$

Concluiu-se que a síndrome de Sheehan associada à miocardiopatia dilatada é rara e não há abordagem terapêutica descrita na literatura. A reposição hormonal para as deficiências apresentadas é o principal recurso disponível conhecido, uma vez que a melhora dos casos clínicos descritos independe da terapia específica para ICC com fração de ejeção reduzida. As principais síndromes tratadas envolvem a reposição do hormônio tireoidiano e corticoterapia, não havendo consenso sobre o benefício da reposição de $\mathrm{GH}{ }^{1}$

\section{Contribuição dos autores}

Concepção e desenho da pesquisa, Obtenção de dados e Análise e interpretação dos dados: Dourado MLBF, Costa TP, Carvalho MS; Análise estatística: Dourado MLBF; Redação do manuscrito: Dourado MLBF, Costa TP; Revisão crítica do manuscrito quanto ao conteúdo intelectual importante: Dourado MLBF, Costa TP, Moura CGG.

\section{Potencial conflito de interesses}

Declaro não haver conflito de interesses pertinentes.

\section{Fontes de financiamento}

O presente estudo não teve fontes de financiamento externas.

\section{Vinculação acadêmica}

Não há vinculação deste estudo a programas de pósgraduação.

\section{Aprovação ética e consentimento informado}

Este artigo não contém estudos com humanos ou animais realizados por nenhum dos autores.

\section{Referências}

1. Soares DV, Conceição FL, Vaisman M. Aspectos diagnósticos e terapêuticos da Síndrome de Sheehan. Arq Bras Endocrinol Metab. 2008; 52(5): 872-7.

2. Snyer PJ. Update. [Internet]. [Cited in 2018 Jun 12] Available from: https://www.uptodate.com/contents/clinical-manifestations-of-hypopi tuitarism? search $=$ hipopituitarismo\&source $=$ search_result\&selectedTi tle $=1 \sim 150 \&$ usage_type $=$ default\&display_rank $=1$

3. Doshi S, Roy A, Ramamoorthy A, Kothari SS, Bahl VK. Dilated Cardiomyopathy. Circ Heart Fail. 2013; 6:e-19-e-21.

4. Organização Pan-Americana da Saúde. (OPAS). Manual de orientação para o curso de prevenção e manejo obstétrico da hemorragia: Zero Morte Materna por Hemorragia. Brasília; 2018.

5. Vieira AEF, Rocha MMD. Síndrome de Sheehan. Relato de caso e revisão da literatura. Rev Bras Med. 2011;14(4):105-8. 
6. Molitch ME. Pituitary diseases in pregnancy. Semin Perinatol. 1998; 22(6):457-70.

7. Islam AKMM, Hasnat MA, Doza F, Jesmin H. Sheehan's syndrome with reversible dilated cardiomyopathy: a case report and brief overview. J Saudi Heart Assoc. 2014; 26(2):117-20.

8. Barisic Júnior S, Barisic GFT, Markman Filho B. Cardiopatias e tireoide. Rev Bras Clin Ter. 2006;63(3):127-33.

9. Natarajan V, Asha HS, Paul T, Sunithi MD. Sheehans syndrome with reversible cardiomyopathy. OA Case Reports. 2014; 3(5):44 .
10. Joshi A, Yonzon I. Sheehan's syndrome associated with reversible dilated cardiomyopathy in na elderly Nepalese woman. Nepal Med Coll. 2015; 17 (3-4):185-6.

11. Ikegami Y, Fukuda T, Jo R, Momiyama Y. Reversible Cardiomyopathy Accompanied by Secondary Adrenal Insufficiency. Circ Heart Fail. 2016; 9(3):e002919.

12. Bhat MH, Bagdadi F, Rafi A Shah PA. Reversible cardiomiopathy as a rare presentation os sheehan's syndrome case report and review of literature. Int J Adv Med. 2017; 4(6): 1713-5. 